\title{
COMPUTATIONAL ANALYSIS OF FLUIDIC COMPRESSION IN SUPERSONIC FLOWFIELDS
}

\author{
Richard G. Haws, ${ }^{*}$ Jeff S. Noall, ${ }^{\dagger}$ and Russell L. Daines ${ }^{\ddagger}$ \\ Department of Mechanical Engineering \\ Brigham Young University \\ Provo, Utah
}

\begin{abstract}
A technique is introduced in which a supersonic flow is compressed in a supersonic inlet by a high pressure jet. This fluidic compression technique is analyzed in the present work using computational techniques. It is shown that by adjusting the pressure of the compression jet, the flow can be kept near design conditions in a fixed-geometry configuration. Results show that this method increases the minimum flowpath area, maintains shock-onlip over a range of Mach numbers, and reduces shock wave/boundary layer interactions. The potential advantages include lower spillage at low Mach numbers, reduced tendency to unstart, and the ability to have near-design operation over a wide range of flight Mach numbers without resorting to variable-geometry machinery. In addition, for combined-cycle engines it yields a low-blockage flowpath for ejector and ramjet modes while maintaining high compression in scramjet mode. Global analysis is used to provide qualitative performance estimates. Grid doubling is used to analyze spatial resolution.
\end{abstract}

\section{INTRODUCTION}

Since the beginning of human flight, the demand for flight vehicles has steadily increased. Today, there is a growing demand for low-cost earthto-orbit and near-orbital flight. Some uses for earth-to-orbit vehicles include satellite deployment and recovery, space station maintenance, military applications, and space tourism. Near-orbital flight offers fast long-distance travel for civilians, as well as advanced reconnaissance and long-range payload delivery for the military.

Rocket engines have been used for years on space-bound vehicles and other vehicles requiring hypersonic speeds; however, rockets deliver low specific impulse, restricting the payload to only a small fraction of the launch weight. Combinedcycle engines are some of the most promising engines for low-cost hypersonic flight. Scramjet engines are a critical stage of combined-cycle engines because, despite their potential a scramjet has not yet been developed which delivers on the promise of high specific impulse. The scramjet, or supersoniccombustion ramjet, engine is an experimental airbreathing engine proposed for use on hypersonic vehicles. Its distinguishing feature is supersonic combustion, which becomes necessary at hypersonic speeds to avoid extreme temperatures in which exothermic reactions are impossible. ${ }^{1}$

\footnotetext{
"Graduate Student, Student Member AlAA

tGraduate Student

*Assistant Professor, Member AIAA

Copyright $@ 1999$ by the American Institute of Aeronautics and Astronautics, Inc. All rights reserved.
}

With the development of scramjet technology, several challenges have arisen. These include achieving efficient compression and efficient air capture over a wide Mach number range, mixing and combustion of fuel, materials, and cooling. The present research is motivated by the need for low spillage at low Mach numbers and high compression at high Mach numbers without variable geometry. The large inlet capture area required for compression at high Mach numbers presents significant challenges at off-design Mach numbers. At transonic speeds, only a small amount of the captured air can be passed through the engine, which can result in high spillage drag. ${ }^{2}$ Consequently, there is a need for engine designs with low spillage requirements; however, the large size of the engine inlet requires much fore-body compression and few moving parts. ${ }^{1}$ Furthermore, compression is limited at high Mach numbers by the shock-induced boundary layer separation.

The current work focuses on fluidic compression of a supersonic air stream. It is shown that fluidic compression can provide inlet starting capability and improve mass capture at Mach numbers below the design Mach number while maintaining high compression ratios at high Mach numbers. It may also prevent shock wave/boundary layer interactions from limiting compression. While the current configuration requires propellant flow rates which outweigh the benefits of higher compression and improved mass capture, alternative configurations may make this technique feasible. In addition, fluidic compression is an alternative form of compres- 


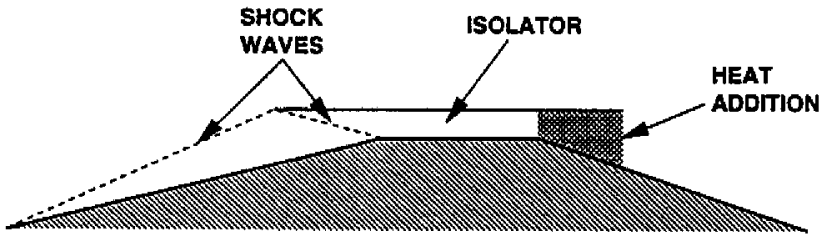

Fig. 1 Schematic diagram of a scramjet engine with a mixed compression inlet.

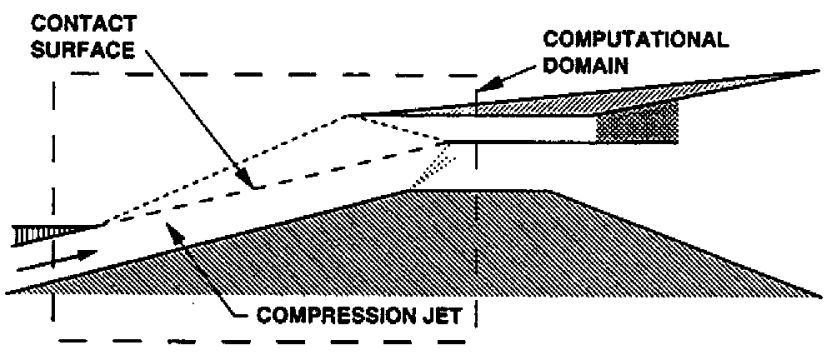

Fig. 2 Schematic of fluidic compression and computational domain.

sion which may prove useful in other applications.

In this work two-dimensional fluidic compression models are developed, and results of the calculated flowfields are presented. The paper first describes fluidic compression and the numerical models used. It then presents the results of the computational analyses, followed by a discussion of conclusions and suggestions for future work.

\section{MODEL DESCRIPTION}

Figure 1 is a schematic of a fixed-geometry scramjet engine with a two-shock, mixed compression inlet. In scramjet operation, incoming air is compressed in the inlet and isolator. The supersonic air then enters the combustor where fuel is added and burned. Following combustion, the gases are expanded and accelerated in a nozzle.

In Fig. 2 fluidic compression is introduced. The compression ramp of Fig. 1 has been replaced by a stream of elevated-pressure gases, which will be called a compression jet. In the present work the compression jet is produced by rocket exhaust. It could also come from sources on board the vehicle, such as fuel or oxidizer. When the air flow and the jet flow meet, they adjust to satisfy the conditions of equal pressure and common flow direction. If the streams are at different pressures initially, compression or expansion waves arise in one or both flows to adjust the pressure as needed. The compression and expansion waves which arise also turn the flow. There is a unique combination of waves which will satisfy the conditions of matched pressure and flow direction.
In the fluidic compression inlet, the jet pressure and flow direction are chosen to be equal to the pressure and flow angle of the inlet air after the desired external shock wave at the design Mach number, as predicted by compressible flow theory. Therefore, at design conditions the jet will remain at constant pressure because of a constantarea flowpath, while the air stream is compressed. Thus, the jet acts as a compression surface, compressing the inlet flow.

At off-design Mach numbers, the same external shock angle is desirable so that the shock extends to the lip of the engine cowl for full air capture. At off-design conditions, however, the air flow direction after this shock will be different than the direction of the jet flow. Therefore, an expansion fan or an oblique compression wave will form in the jet, emanating from the point where the two streams meet. The wave will reflect off the lower jet wall and affect the air stream. These effects must be accounted for when choosing the jet pressure which will locate the external shock on the cowl lip. For full inlet mass capture, the jet pressure will vary as the flight Mach number changes. Since the jet is directly injected, the injection pressure can be directly controlled.

The virtual compression surface created by the compression jet ends at a splitter plate. In order to minimize the physical contraction ratio, the lower jet wall is turned horizontal as soon as possible without affecting the upper stream. The splitter plate prevents the expansion fan in the jet from entering the air flow. It also prevents the internal shock wave in the air flow from compressing the jet and separates the jet from the compressed inlet air stream so that fuel can be burned in the air stream. Both the air stream and the jet are expanded in a nozzle, producing thrust.

The benefits of fluidic compression are improved off-design mass capture and high achievable compression ratios in a startable, fixed-geometry engine. Off-design mass capture is improved through the ability to control the position of the external shock and maintain the shock-on-lip condition over a range of Mach numbers. One of the greatest potential benefits of fluidic compression, however, is that it decreases the physical area contraction necessary to achieve high internal compression. When the compression jet is not flowing, the flowpath is less obstructed than a solid-wall inlet with the same degree of internal compression. This makes it possible to start the inlet. Then, when internal supersonic flow has been established, the jet can be introduced for maximum compression. 
By using fluidic compression, the problem of engine unstart from the internal shock wave causing boundary layer separation is avoided. At the point where the jet and the inlet air stream meet, boundary layers exist immediately upstream of the contact point. As the shear layer develops between the air stream and the compression jet, the lowmomentum fluid from the boundary layers is accelerated. By the time this flow encounters the splitter plate, the velocity is supersonic and exhibits a smooth transition from the air stream velocity and the jet velocity. Any boundary layer separation caused by the internal shock wave cannot propagate upstream of the splitter plate to cause unstart; thus, fluidic compression can increase the amount of internal compression achievable without boundary layer bleed.

There are also drawbacks associated with fluidic compression due to the presence of a splitter plate and the increased propellant used to produce the jet flow. One of the difficulties associated with the splitter plate is that the optimum location of the splitter plate changes with varying Mach number. At the optimum location, the splitter plate performs the following three functions:

1. The splitter plate separates the compressed air from the compression jet.

2. It prevents the expansion fan from the lower jet wall from expanding the compressed air.

3. The plate intercepts the internal shock wave which emanates from the cowl lip, preventing shock losses in the jet flow.

All three functions can only be performed at a single flight Mach number when the shear layer, the internal shock, and the expansion from the lower wall all intersect at a single point. This presents two options in designing the splitter plate location: a translating splitter plate to optimize performance or a stationary splitter plate. The motion of a translating splitter plate would be limited to sliding along a predetermined path. Even this simple motion, however, would require moving parts and increase the vehicle weight. Furthermore, translating the splitter plate can, in general, only optimize two of the three functions it performs because it is unlikely that the shock, shear layer, and expansion intersect at a single point at offdesign conditions.

The location of a fixed splitter plate would depend on the relative importance of several offdesign loss mechanisms. At low Mach numbers, the jet will be compressed by the inlet air. This will decrease the cross-sectional area of the jet streamtube and cause some of the inlet air to flow around the splitter plate and not enter the combustor. At high Mach numbers, the jet will expand, allowing some of the jet gases to flow around the splitter plate and mix with the air flow. In addition, the shock from the inlet cowl could enter the jet flow at some conditions, causing shock losses, while at other conditions the expansion fan could enter the air flow, decreasing compression.

There are other drawbacks associated with any splitter plate. The presence of a splitter plate increases the wetted area, causing increases viscous losses. Furthermore, a physical splitter plate will have finite thickness which will cause shocks to form and increase shock losses. The leading edge of the splitter plate also presents significant cooling challenges. Further research is required in all these areas.

While providing significant benefits, the jet flow itself has penalties associated with it. All of the jet flow comes from on-board fuel, oxidizer, or both. The specific impulse of the jet flow will be less than the specific impulse of the main flow. The jet flow may also experience shock losses when the splitter plate ends and the flows again join. In order for fluidic compression to be feasible, the improved performance of the main flow and the weight reduction from the use of a fixed-geometry inlet must offset these penalties.

\section{SOLUTION TECHNIQUE}

As seen in Fig. 2, the computational domain begins slightly upstream of where the jet is introduced and extends downstream of the leading edge of the splitter plate. Modeling of fluidic compression is accomplished using a diagonalized $\mathrm{ADI}$ algorithm, ${ }^{3}$ the $q-\omega$ turbulence model of Coakley, ${ }^{4}$ inviscid and viscous time step preconditioning, and a TVD scheme to resolve shocks. In order to limit the computational time, all models are twodimensional. Because of the expected importance of boundary layers, the computations include the effects of turbulence and viscous walls. The air and jet flow inlets are supersonic, with the boundary conditions specifying Mach number, flow angle, species concentrations, and static temperature and pressure. Exits are supersonic, with flowfield properties extrapolated from inside the computational domain.

The air inlet conditions are obtained as follows. Free-stream conditions are obtained from a trajectory analysis for a representative engine. ${ }^{5} \mathrm{~A}$ 
Table 1 Free-stream conditions and air inlet boundary conditions to the computational domain after the assumed fore-body shock.

\begin{tabular}{|ccc|ccc|}
\hline \multicolumn{3}{|c|}{ Free-Stream } & \multicolumn{3}{c|}{ Air Inlet } \\
$M$ & $P(\mathrm{kPa})$ & $T(\mathrm{~K})$ & $M$ & $P(\mathrm{kPa})$ & $T(\mathrm{~K})$ \\
\hline \hline 4.7 & 6.55 & 217 & 4.0 & 14.8 & 277 \\
6.0 & 4.46 & 219 & 5.0 & 12.3 & 302 \\
9.2 & 2.08 & 224 & 7.0 & 8.92 & 370 \\
\hline
\end{tabular}

fore-body half angle of 7.5 degrees is assumed, and the pressure and temperature at the inlet are calculated from shock theory, assuming a calorically perfect gas with a specific heat ratio of 1.4. Three flight Mach numbers are studied: 4.7, 6.0, and 9.2, which correspond to Mach numbers at the beginning of the inlet of $3.0,5.0$, and 7.0 , respectively. Table 1 lists the air inlet boundary conditions for the three Mach numbers studied.

Three compression jet Mach numbers are studied, as well as two jet exit areas. The jet pressure in each case is chosen to locate the external shock on the cowl lip. The stagnation temperature of the jet comes from a typical rocket combustion chamber at $3200 \mathrm{~K}(5760 \mathrm{R})$, and the jet is specified as gaseous $\mathrm{H}_{2} \mathrm{O}$. The jet enters at an inclination of 14 degrees to the air flow.

Two grids are used, with jet widths of 0.271 and 0.181 times the width of the nominal inlet capture area. Grid points are clustered near solid-wall boundaries, and for many of the cases the grid is adapted to cluster points near high gradients in pressure and velocity. The grid dimensions are 450 points in the cross-stream direction and 606 points in the stream-wise direction. This work is completed in support of an experimental program which has not yet been carried out. For this reason, the portion of the grid upstream of the engine cowl is extended vertically, topped by a solid wall boundary condition (see Fig. 3). The extended region provides sufficient space for removal of the boundary layer to simulate free-stream conditions at the cowl, as seen by the absence of boundary layer effects at the cowl lip.

A global analysis is also performed on the fluidic compression model. In this analysis, the parameters of the compression jet are varied to determine their effect on the jet flow rates required for fluidic compression. This is done over a range of vehicle Mach numbers along the vehicle trajectory. A specific impulse of $2000 \mathrm{~s}$ is assumed for the scramjet, and the lower jet is assumed to be a rocket with a specific impulse of $428 \mathrm{~s}$. Using the mass flow rates calculated for the inlet air and for the compression jet, the total specific impulse is calculated. This information is used to evaluate the effect of the jet Mach number and the jet width on the total specific impulse at different flight Mach numbers.

\section{RESULTS}

One of the potential benefits of fluidic compression is that it decreases the physical area contraction necessary to achieve high internal compression. When the compression jet is not flowing, the flowpath is less constricted than a solid-wall inlet with the same degree of internal compression. This makes it possible to start the inlet. Once internal supersonic flow is established, introducing the jet flow increases the contraction ratio of the inlet streamtube without variable geometry.

A two-shock, mixed-compression inlet with a 14degree compression ramp, designed for operation at Mach 5, has an overall contraction ratio of 3.90 . With fluidic compression, the minimum physical area is increased to include the region below the splitter plate. With the compression jet on, this inlet still yields the higher air-stream contraction ratio of the mixed compression inlet. When the jet is off, however, the small physical contraction ratio can allow for greater mass capture at low Mach numbers. Small physical contraction ratios can also improve the performance of combined-cycle engines during ejector and ramjet modes. The jet widths studied result in physical contraction ratios as low as 1.98 for the smaller jet and 1.59 for the wider jet, assuming the expansion in the compression jet just touches the leading edge of the splitter plate as in Fig. 2. In this work, however, turning of the lower jet wall to horizontal is delayed until directly below the leading edge of the splitter plate, as seen in the grid in Fig. 3, in order to ensure that the expansion does not affect the air flow. This results in smaller minimum flowpath area and a higher contraction ratio. The physical contraction ratios for the cases studied are 2.26 and 1.87 for the narrow jet and the wide jet, respectively.

Several important conclusions can be drawn from the global analysis. Figure 4 shows the effect of changing the jet area and jet Mach number on the jet flow rate. The jet mass flow scales directly with jet area and with jet Mach number at all flight Mach numbers; however, as the jet area decreases the engine flowpath is constricted, jeopardizing low Mach number starting capability. This indicates that the jet flow rate can be most effectively reduced by reducing the jet Mach number, while keeping an open engine flowpath.

Figure 5 shows the total specific impulse for dif- 


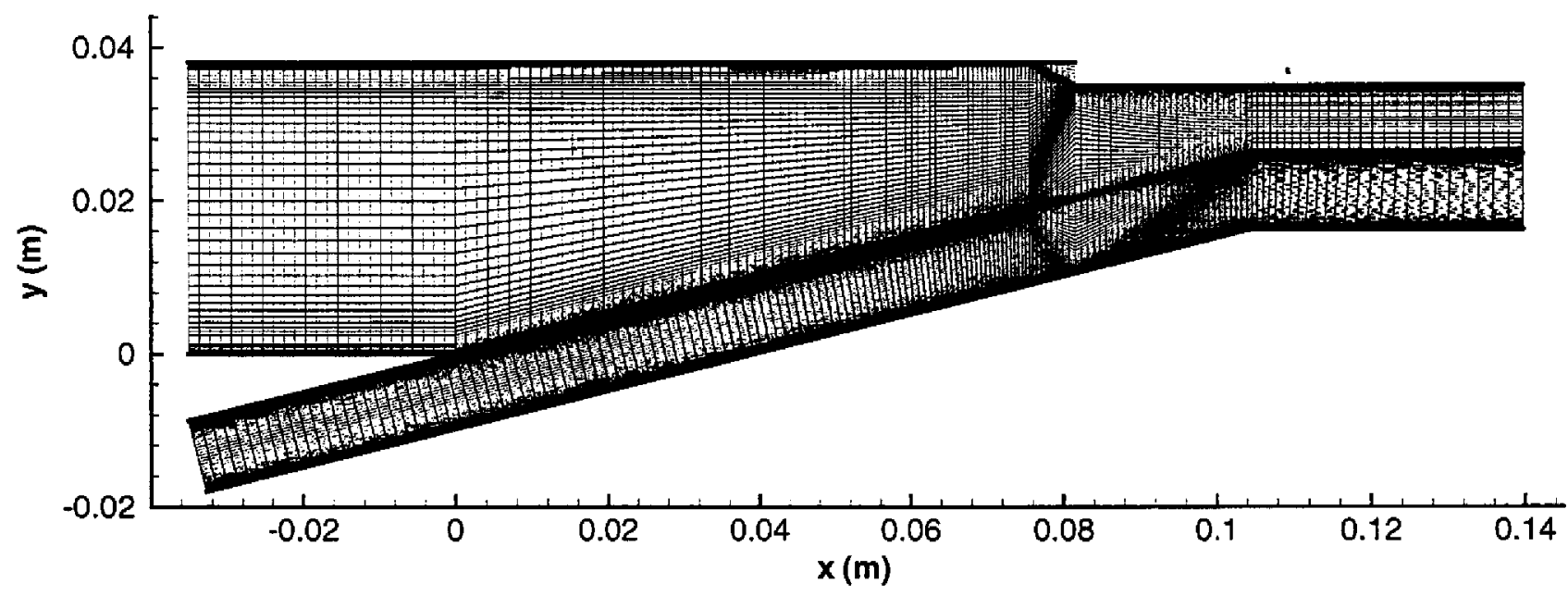

Fig. 3 Computational grid for the fluidic compression model with a jet to capture area ratio of 0.271.

ferent jet Mach numbers along the vehicle trajectory, assuming full mass capture. With the assumed values of specific impulse for the air flow and the compression jet, the total specific impulse becomes an average of the two values, weighted by mass flow. Therefore, as the jet flow rate approaches zero, the total specific impulse approaches the specific impulse of a variable-geometry scramjet with full mass capture. This figure shows the importance of minimizing the jet flow. If we can keep the mass flow of the jet to less than $25 \%$ of that of the mass flow rate of the fuel for the air stream then fluidic compression will be beneficial or neutral in specific impulse, but engine weight will have been significantly decreased with fixedgeometry fluidic compression.

To obtain flowfield details and to enhance understanding of the fluidic compression process, full computational fluid dynamic solutions are obtained. The results of this analysis demonstrate fluidic compression with full mass capture over a range of Mach numbers. Unless otherwise stated, all plots are for a jet Mach number of 2.0 and a jet to capture area ratio of 0.271 . Computations are also successfully carried out at Mach 6.0 for jet Mach numbers of 1.1 and 3.0 with the same geometry, and with a jet Mach number of 2.0 with a smaller jet $(0.181$ jet to capture area ratio), with similar results.

Figures. 6 and 7 show the Mach number and pressure fields at the design Mach number of 6.0. The free-stream pressure is $4.46 \mathrm{kPa}(0.647 \mathrm{psia})$. After the assumed fore-body shock, the air enters the computational domain at Mach 5.0 and

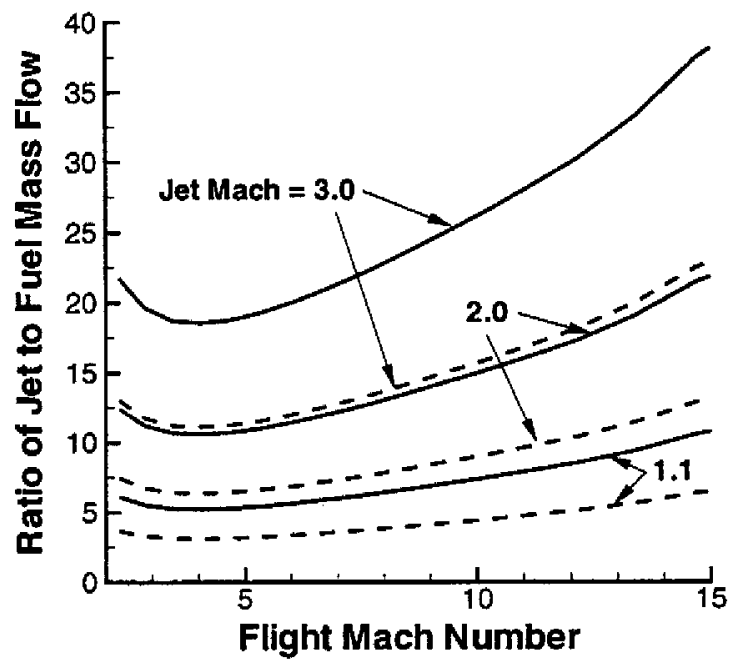

Fig. 4 Ratio of jet flow rate to (stoichiometric) scramjet fuel flow rate required for fluidic compression using a gaseous $\mathrm{H}_{2} \mathrm{O}$ jet with jet area to capture area ratios of 0.181 (solid lines) and 0.271 (dashed lines).

$12.3 \mathrm{kPa}$ (1.78 psia). Across the external shock the Mach number is reduced to 3.61 , while the pressure rises to about $59 \mathrm{kPa}$ ( $8.6 \mathrm{psia}$ ) to match the pressure of the compression jet. The internal shock further reduces the Mach number to 2.79, and the final pressure is about $180 \mathrm{kPa}$ (26 psia) entering the isolator. The overall static pressure ratio from free stream to the isolator is about 40 . The jet entrance Mach number is specified as 2.0, and as it enters the air flow, it is flowing at Mach 1.94, with a pressure of about $60 \mathrm{kPa}(8.7 \mathrm{psia})$. 


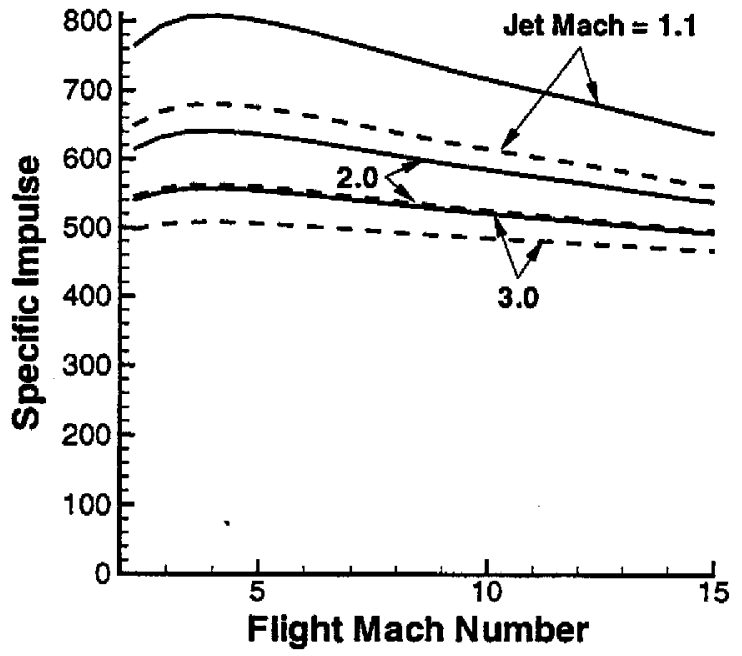

Fig.5 Total specific impulse for jet area to capture area ratios of 0.181 (solid lines) and 0.271 (dashed lines). Scramjet and compression jet specific impulse are constant.

In Figs. 8 and 9, the Mach numbers and pressures are shown for a flight Mach number of 4.7 and a free-stream pressure of $6.55 \mathrm{kPa}(0.950 \mathrm{psia})$. After the fore-body shock, the inlet Mach number is 4.0 , and the pressure is $14.6 \mathrm{kPa}$ (2.12 psia). The external inlet shock reduces the Mach number to 3.1 and increases the pressure to $54 \mathrm{kPa}$ (7.83 psia). The final Mach number as the air enters the isolator is 2.4 , and the pressure is $150 \mathrm{kPa}(22 \mathrm{psia}$ ) for a compression ratio of 23 .

At free-stream conditions of Mach 9.2 and $2.08 \mathrm{kPa}(0.302 \mathrm{psia})$, the inlet flow enters at Mach 7.0 and $8.92 \mathrm{kPa}$ (1.29 psia). The calculated Mach number and pressure fields are shown in Figs. 10 and 11. The external shock brings the flow to Mach 4.5 and about $65 \mathrm{kPa}$ (9.4 psia), and the internal shock further reduces the Mach number to about 3.4 and raises the pressure to $260 \mathrm{kPa}$ (38 psia). The overall pressure ratio is 125 .

Figures. 7, 9, and 11 show that fluidic compression enables the engine to maintain the shock-onlip condition over a range of Mach numbers for increased mass capture at low Mach numbers and better performance at high Mach numbers, by judiciously choosing the jet pressure. The external shock in the Mach 6.0 case, seen in Fig. 7, falls near the lip of the inlet cowl; this was expected because the geometry is designed for this Mach number. This allows for full mass capture at the design Mach number. The external shock in the Mach 4.7 case, shown in Fig. 9, demonstrates shock control below the design Mach number. Figure 11 shows that the external shock can also be properly placed at a flight Mach number of 9.2. This suggests that the shock can also be controlled at over-speed conditions for better performance.

The location of the internal shock wave in relation to the splitter plate changes with flight Mach number. Figure 12 shows that at a Mach number of 4.7, the internal shock is near the edge of the splitter plate. At Mach 6.0, the shock extends further into the isolator region (Fig. 13), and further at Mach 9.2 (Fig. 14). Figures. 12-14 also show the variation of where the internal shock would intersect the shear layer in the absence of a splitter plate. The small variation shows that a fixed splitter plate would provide good performance over this entire range of Mach numbers. Extension of this work to higher Mach numbers is needed to determine the effect of higher Mach numbers.

When the internal shock wave lands behind the leading edge of the splitter plate, it causes a small separation region between the plate edge and the shock (see Fig. 15); however, the separation cannot propagate further upstream. This reduces the risk of unstart from shock-induced boundary layer separation at high contraction ratios.

To determine the adequacy of the grid spacing for the solutions presented above, a grid doubling study was conducted. It was performed on the baseline case, which had an inlet Mach number of 5.0 and a wide compression jet with a Mach number of 2.0. The grid was doubled in both the axial and cross-stream directions. Profiles of axial velocity are shown in Fig. 16 for an axial location $5 \mathrm{~mm}$ ( $0.127 \mathrm{in})$ downstream of the leading edge of the splitter plate. This was the location that exhibited the largest error. It can be seen that there is good agreement between the two solutions. The largest error is approximately $70 \mathrm{~m} / \mathrm{s}$, occurring about $3.5 \mathrm{~mm}(0.089 \mathrm{in})$ above the lower wall. Using Richardson's extrapolation, this corresponds to a maximum error on the baseline grid of $3.4 \%$. Throughout the majority of the flowfield the error is less than $1 \%$. From this it is concluded that the grid spacing used in these cases is sufficiently small that numerical resolution errors are not significant. This grid doubling study was conducted using a non-adapted grid; however, for many of the cases the grid was adapted to cluster points near high gradients in pressure and velocity and increase the numerical accuracy. 


\section{CONCLUSIONS}

Fluidic compression has been analyzed using computational fiuid dynamic models. In these models, a jet of high pressure gas injected at an angle to the main air flow is shown to compress supersonic air, with the contact surface between the air and the jet forming a virtual compression ramp. The numerical accuracy of the models is verified through a grid doubling study. A main conclusion from this work is that by adjusting the pressure of the compression jet, the flow can be kept near design conditions in a fixed-geometry configuration.

By using fluidic compression, the minimum area of the flowpath can be increased compared to geometric compression. In the cases presented, the capture streamtube is compressed with an area contraction ratio of 3.90 , while the geometric contraction ratio is either 2.26 or 1.87 , depending on the jet discharge area. The flowpath could be opened further with an optimized jet flowpath. For example, with a jet to capture area ratio of 0.271 , the contraction ratio is as low as 1.59 , yielding a minimum area which is 2.45 times greater for fluidic compression than for geometric compression.

Fluidic compression is also shown to allow full air mass capture over a range of Mach numbers without variable geometry. Full air capture is demonstrated at flight Mach numbers of 4.7,6.0, and 9.2 for an inlet system designed for Mach 6.0. Full mass capture comes at the cost of increased propellant flow rates. The jet flow should be minimized in order to minimize the additional propellant needed. The results of a global analysis suggest that this can be accomplished most effectively by reducing the Mach number of the compression jet.

Future work in the area of fluidic compression should focus on quantifying the benefits of fluidic compression and minimizing the propellant flow rate required to produce the compression jet. Work to quantify the benefits would include modeling a complete fluidic compression engine and comparing its performance to the performance of a conventional fixed-geometry engine. One useful comparison would be between engines with the same air contraction ratio. Another would be to determine how much the startable contraction ratio can be increased compared to current high contraction ratio, fixed-geometry engines. Reductions in compression jet flow rates may be realized by using a gas with a low molecular weight and reducing the jet Mach number.

\section{ACKNOWLEDGMENTS}

This work was supported by the NASA Lewis Research Center under contract NAS3-97020. The authors would like to thank Dick Foster and Fred Billig for their input in this work.

\section{REFERENCES}

${ }^{1}$ Ferri, A., "Mixing-Controlled Supersonic Combustion," Annual Review of Fluid Mechanics, vol. 5, 1973, pp. 310-308.

${ }^{2}$ Leynaert, J., Surber, L., and Goldsmith, E., Practical Intake Aerodynamic Design University Press, Cambridge, 1993, ch. 5.

${ }^{3}$ Buelow, P. E., Schwer, D. A., Feng, J., Merkle, C. L., and Choi, D., "A Preconditioned Dual-Time, Diagonalized ADI Scheme for Unsteady Computations," AIAA Paper 97-2101, 13th AlAA Computational Fluid Dynamies Conference.

${ }^{4}$ Coakley, T., "Turbulence Modeling Methods for the Compressible Navier-Stokes Equations," ALAA Paper 83-1693, AIAA 16th Fluid and Plasma Dynamics Conference, Danvers, Massachusetts.

${ }^{5}$ Olds, J., and Bradford, J., "SCCREAM (Simulated Combined Cycle Rocket Engine Analysis Module): A conceptual RBCC Engine Design Tool," AIAA Paper 97-2760, 33rd AIAA/ASME/SAE/ASEE Joint Propulsion Conference, Seattle, WA. 


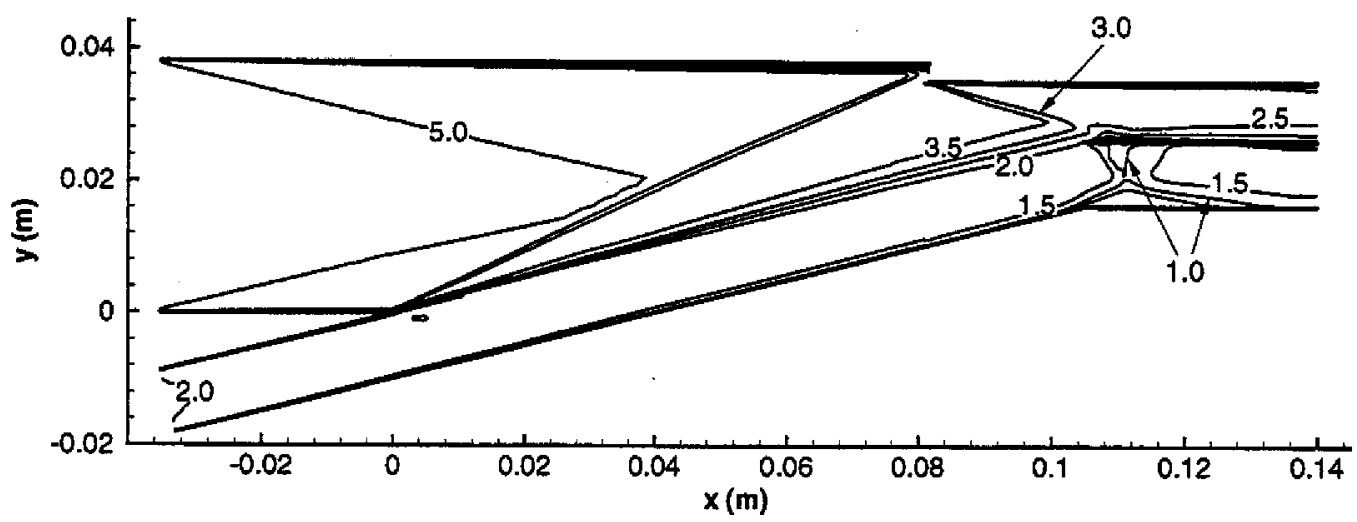

Fig. 6 Mach number contours of the fluidic compression model at a flight Mach number of 6.0 with a jet to capture area ratio of 0.271 and a jet Mach number of 2.0 .

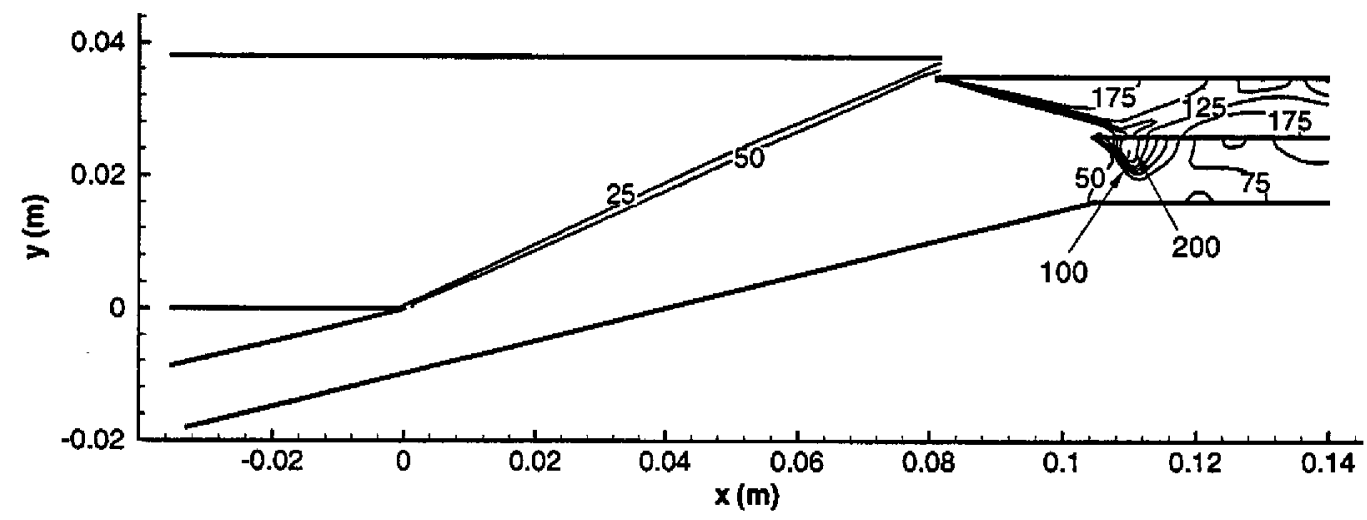

Fig. 7 Pressure contours of the fluidic compression model at a flight Mach number of 6.0 with a jet to capture area ratio of 0.271 and a jet Mach number of 2.0.

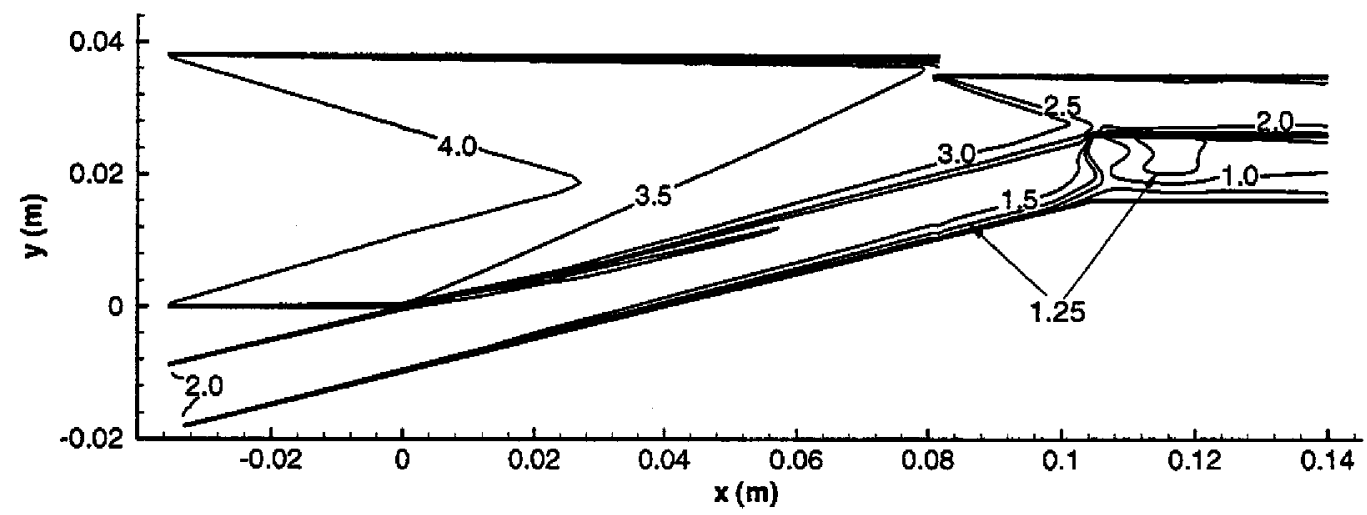

Fig. 8 Mach number contours of the fluidic compression model at a flight Mach number of 4.7 with a jet to capture area ratio of 0.271 and a jet Mach number of 2.0 . 


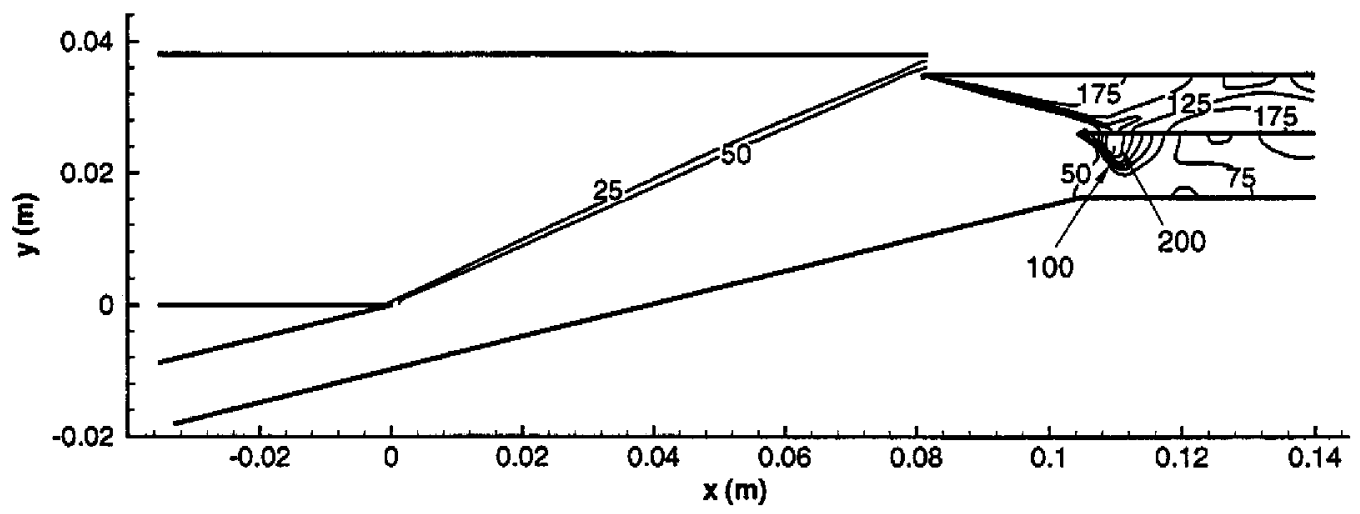

Fig. 9 Pressure contours of the fluidic compression model at a flight Mach number of 4.7 with a jet to capture area ratio of 0.271 and a jet Mach number of 2,0.

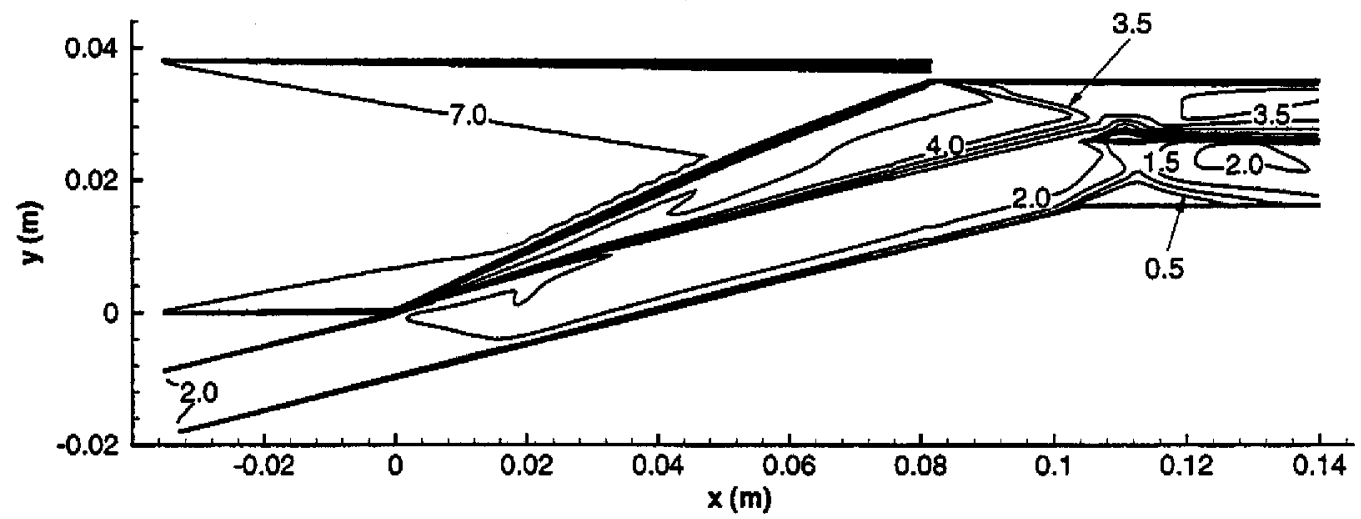

Fig. 10 Mach number contours of the fluidic compression model at a flight Mach number of 9.2 with a jet to capture area ratio of 0.271 and a jet Mach number of 2.0.

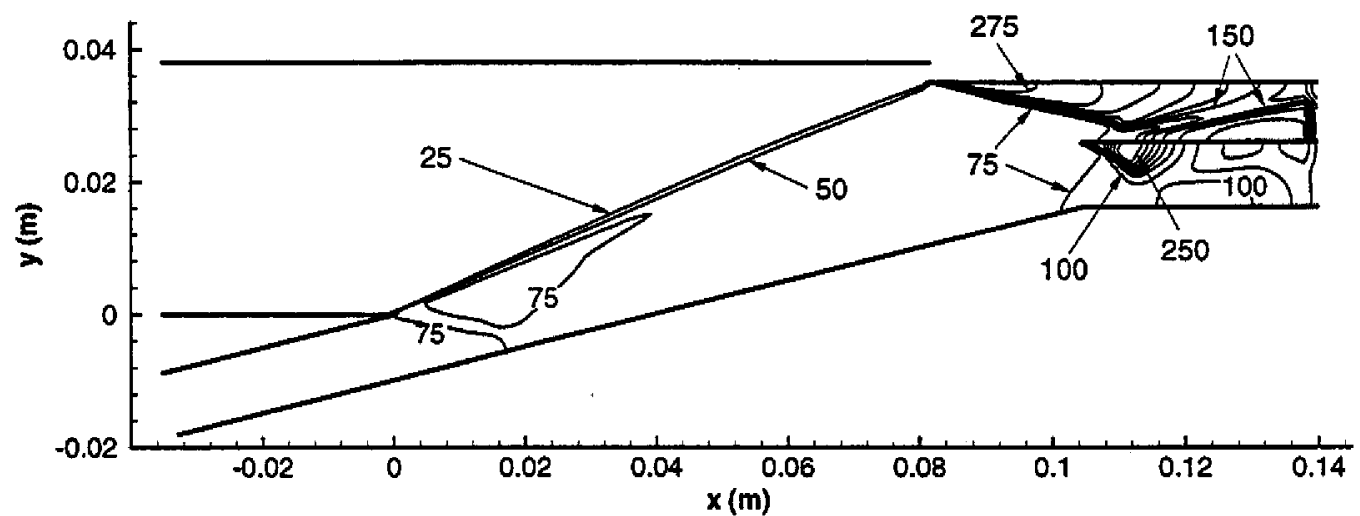

Fig. 11 Pressure contours of the fluidic compression model at a flight Mach number of 9.2 with a jet to capture area ratio of 0.271 and a jet Mach number of 2.0. 


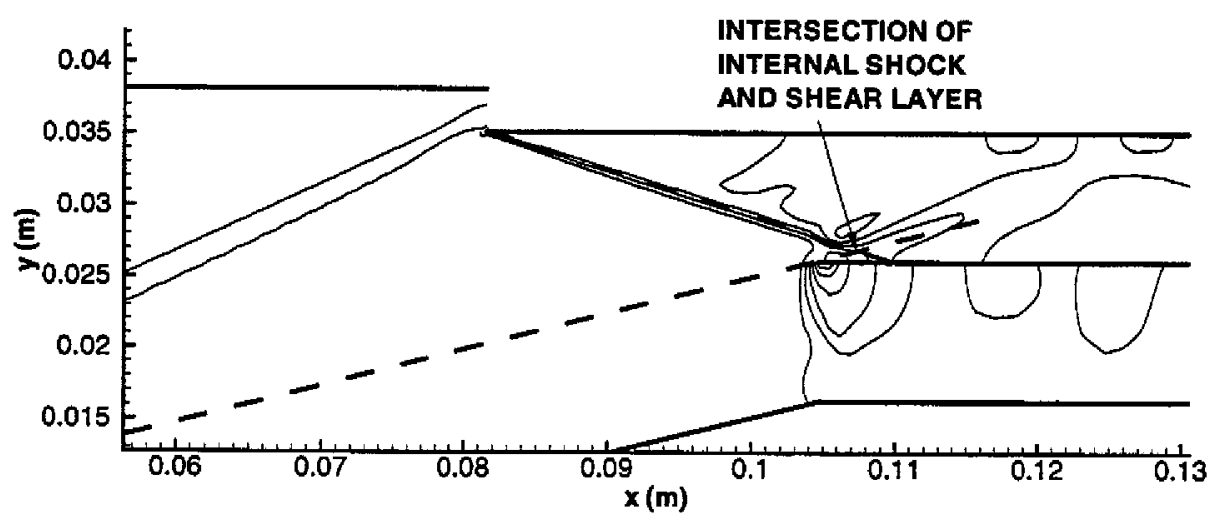

Fig. 12 Pressure contours in the region of the internal shock wave at a flight Mach number of 4.7. The shock wave intersects the shear layer at grid coordinates $(0.107,0.027)$.

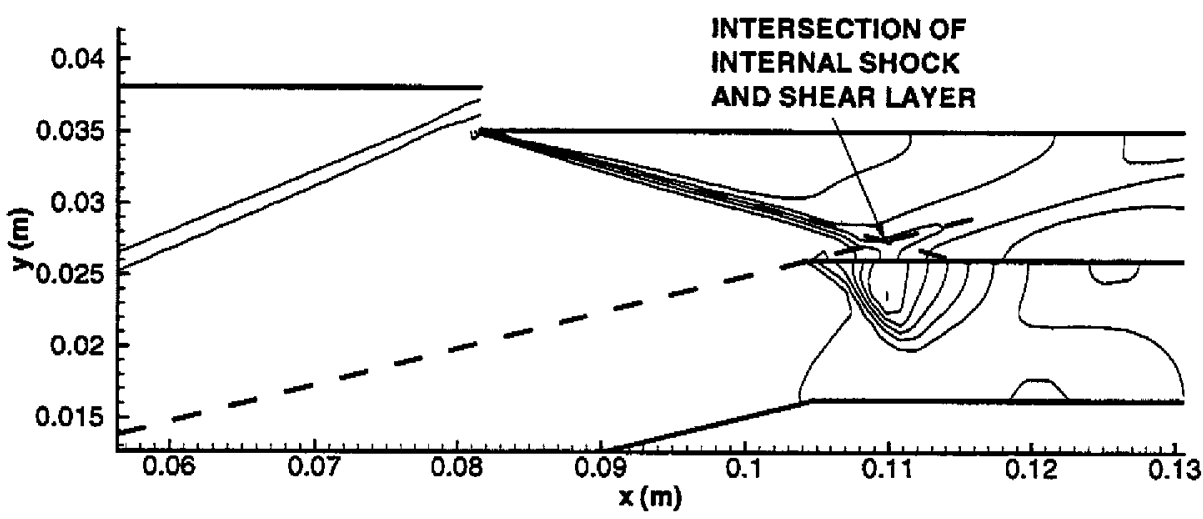

Fig. 13 Pressure contours in the region of the internal shock wave at a flight Mach number of 6.0. The shock wave intersects the shear layer at grid coordinates $(0.110,0.028)$.

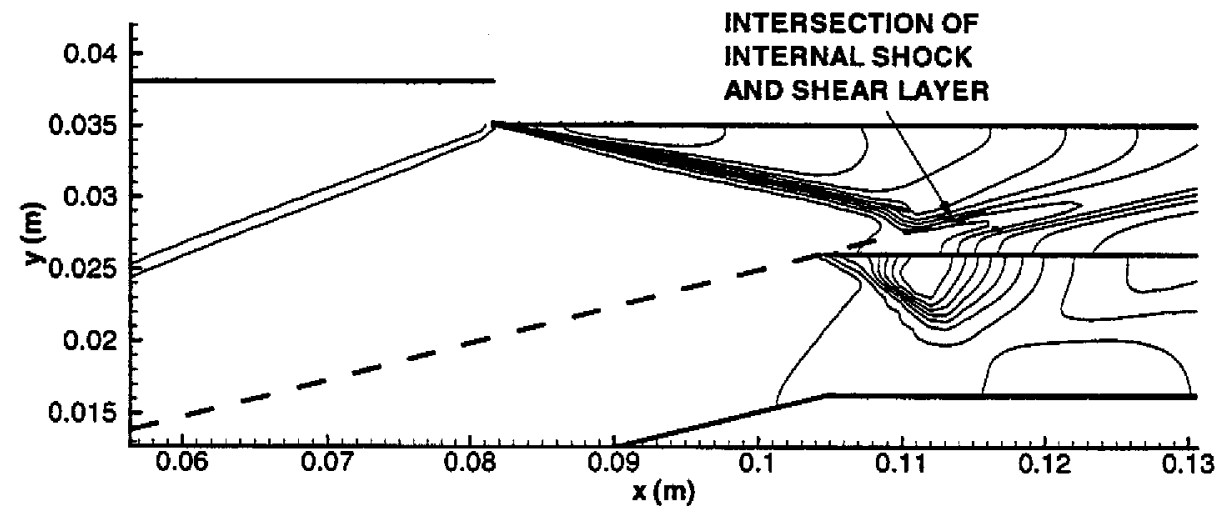

Fig. 14 Pressure contours in the region of the internal shock wave at a flight Mach number of 9.2. The shock wave intersects the shear layer at grid coordinates $(0.114,0.029)$. 


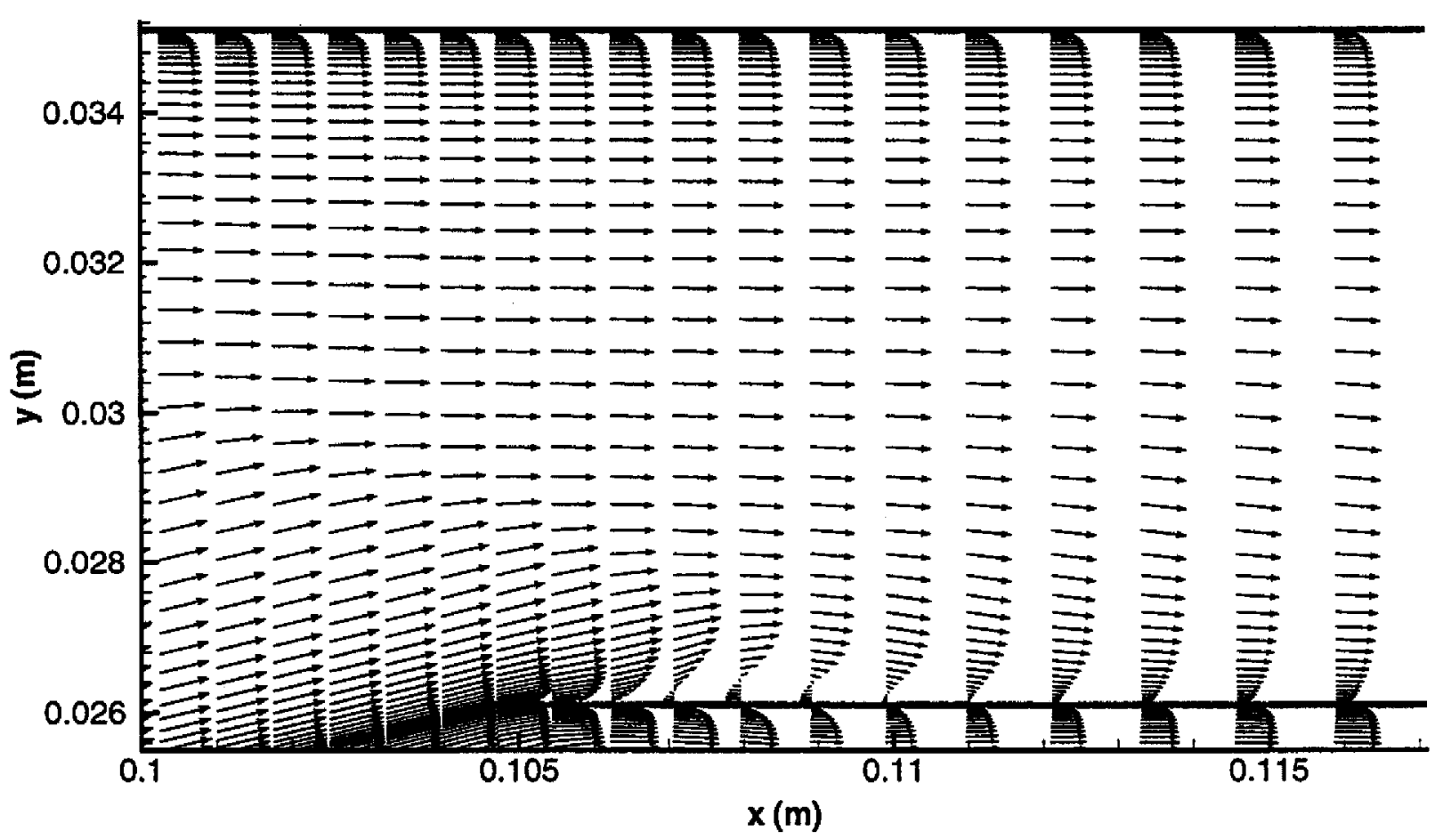

Fig. 15 Velocity vectors near the splitter plate leading edge at a flight Mach number of 9.2.

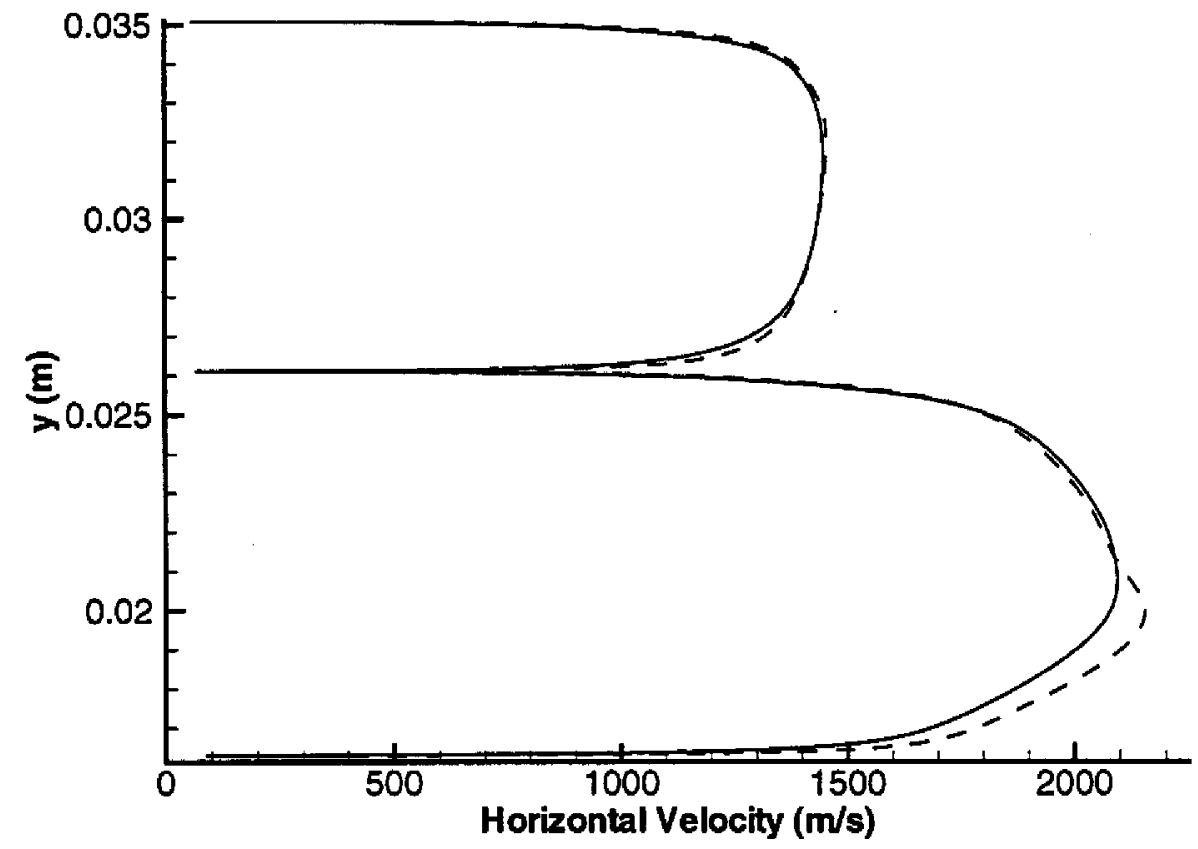

Fig. 16 Axial velocity profile comparison for the baseline grid (solid line) and a doubled grid (dashed line) at an axial location $5 \mathrm{~mm}(0.127 \mathrm{in})$ downstream of the leading edge of the splitter plate. The maximum exror of $3.4 \%$ occurs $3.5 \mathrm{~mm}(0.089 \mathrm{in})$ above the location of the lower wall. 